Literarische Geselischaft s. Sprachgesellschaft

\title{
Literarische Institutionen
}

1. Begriff

2. Gesellschaftliche Grundlagen

3. Konstituenten des Literatursystems

4. Historische Entwicklung

\section{Begriff}

Der Begriff L. I. hat zwar Eingang in die Alltagssprache gefunden, verdankt sich jedoch einer Reihe von nicht auf den dt.sprachigen Raum konzentrierten neueren Entwicklungen innerhalb der Literaturwissenschaft, deren Wurzeln in der seit den späten 1960er Jahren immer deutlicheren Hinwendung zur Soziologie als neuer Leitdisziplin zu finden sind. Im Zuge einer zunehmend sozialgeschichtlich ausgerichteten Beschäftigung mit 7 Literatur interessierte nun nicht mehr primär das poetische Kunstwerk als solches und die mit diesem verbundene hermeneutische Herausforderung, sondern auch das System jener I., welche die Herstellung (Produktion), Verteilung (Distribution), Vermittlung und Lektüre (Rezeption) lit. Werke überhaupt erst ermöglichen. Im Fokus standen dabei von Anfang an zum einen die vielfältigen Akteure und Praktiken, die für die lit. Kommunikation als konstitutiv zu gelten haben, und zum anderen der Zusammenhang zwischen dem, was man mit Pierre Bourdieu als mlit. Feld " bezeichnen kann [2], und anderen Feldern gesellschaftlichen Handelns, wie etwa Politik, Okonomie, Religion oder Bildungswesen. 
Parallel zum Geltungsgewinn einer sozialgeschichtlichen Perspektive, die in den $1980 e r$ Jahren den literaturwiss. Diskurs beflügelte und phasenweise dominierte, fand auch der Begriff L.I. zunehmende Verbreitung, ohne allerdings jene definitorische Prägnanz zu erreichen, die es erlauben würde, von einem Schlüsselbegriff literaturwiss. Analyse zu sprechen [3]; [4]; [5]. Denn L. I. können, je nach Verwendungszusammenhang, Unterschiedliches bezeichnen; der Begriff steht außerdem in Konkurrenz zu bisweilen synonym benutzten Termini wie "lit. Kommunikationssystem “, »lit. Markt " oder "lit. Leben" [6].

Die Schwierigkeit, den Terminus L. I. präzise zu fassen, ergibt sich nicht zuletzt daraus, dass er sich aus zwei Wörtern zusammensetzt, die ihrerseits sehr unterschiedliche Auslegungen erfahren haben. So besteht mit Blick auf "Literatur « kein Konsens darüber, was deren Objektbereich ausmacht. Beschränken sich die Verfechter eines engen Literaturbegriffs auf jene begrenzte Zahl poetischer Werke, deren besondere ästhetische Qualität die Aufnahme in einen jeweils historisch spezifischen, jedoch meist mit Anspruch auf überzeitliche Geltung antretenden $/$ Kanon rechtfertigen, so erweitern die Exponenten eines weiten Literaturbegriffs das Feld auf sämtliche sich der Sprache bedienenden und einer oder mehreren 7 Gattungen zuzuordnenden schriftlichen Manifestationen, zu denen auch Sachliteratur wie etwa gelehrte oder geistliche Prosa gehören kann.

Im Folgenden meint "Literatur « allerdings weder ein überschaubares Ensemble in ästhetischer Hinsicht herausragender Werke noch einen Sammelbegriff für jede Form schriftlichen Ausdrucks, sondern die Gesamtheit jener Texte, die das Merkmal der »Literarizität" erfüllen. Diese ist dort gegeben, wo in einem Text jene beiden Konventionen dominieren, die als Ästhetik- bzw. Polyvalenz-Konvention bezeichnet werden [8.103-143].

Ästhetik-Konvention bedeutet, dass ein lit. Text nicht in erster Linie danach beurteilt werden will, ob das, was er beschreibt, wahr oder falsch ist, sondern danach, ob es poetisch gerechtfertigt werden kann. Nicht der - durch ein in einem bestimmten histor. Moment jeweils geltendes Wirklichkeitsmodell begründete Wahrheitsgehalt, sondern der ästhetische Charakter entscheidet demnach über den lit. Status eines Textes. „Ästhetisch" verweist in diesem Zusammenhang weniger auf ein Werturteil als vielmehr ganz grundsätzlich auf die Intention eines Textes und ist deshalb auch mit Blick auf all jene lit. Manifestationen gegeben, die von der Thiteraturkritik der sog. Trivialliteratur zugeordnet wurden. Polyvalenz-Konvention wiederum steht für die Freiheit der Urheber lit. Werke, einem Text unterschiedliche Deutungsangebote einzuschreiben und ihn so anzulegen, dass er den 7 Lesern Interpretationsspielräume eröffnet, statt sie auf eine einzige Lesart festzulegen.
Leitend ist demnach ein weiter Begriff von Literatur, der poetische Texte unterschiedlichen ästhetischen Rangs umfasst und zugleich berücksichtigt, dass Literarizität "nur historisch-pragmatisch definierbar" ist, da die Interpretation sowohl der Ästhetik- als auch der Polyvalenz-Konvention durch die in einem histor. spezifischen sozio-kulturellen Umfeld agierenden Protagonisten des Literatursystems darüber entscheidet, »welche Objekte als lit. eingeschätzt und behandelt werden « [8.14]

Nicht weniger klärungsbedürftig als der Begriff Literatur ist jener der Institution. Der innerhalb der Soziologie gleichermaßen intensiv und kontrovers diskutierte Terminus ist durch eine doppelte Konnotation gekennzeichnet: I. stehen zum einen für Normen und Werte, die soziales Handeln determinieren, und zum anderen für die materiellen Träger dieser Normen und Werte bzw. für jene informellen und formellen Organisationen, denen mit Blick auf ihre Verfestigung, Durchsetzung und Sanktionierung zentrale Bedeutung zukommt. Zu beachten gilt auch hier, dass I. ihre konkrete Ausformung und ihren gesellschaftlichen Status jeweils spezifischen sozio-kulturellen und histor. Bedingungen verdanken, dass sie also in engem Zusammenhang mit jeweils zeitgenössischen Gegebenheiten gesehen werden müssen sowie histor. Wandlungsprozessen unterworfen sind. Man hat daher auch gefordert, dass "zwei Dimensionen institutioneller Analyse" sich ergänzen müssten: weine synchronische als Erörterung institutioneller Strukturzusammenhänge und eine diachronische als Ort der Erfassung jener histor. Besonderheit, in der die abstrakten Erwägungen der ersten Ebene einzulösen ... $\operatorname{sind}$ " $[7.113 \mathrm{f}$.$] .$

\section{Gesellschaftliche Grundlagen}

Ausgehend von diesen terminologischen Klärungen lassen sich L.I. demnach als Ensemble gesellschaftlich festgelegter und kodifizierter Normen bestimmen, welches die Produktion, Distribution, Vermittlung und Rezeption als lit. definierter Texte determiniert; zugleich lassen sie sich auch als die institutionellen Träger verstehen, welche diese Vorgänge organisieren. Dem Bereich der Normen sind dabei nicht nur jene ästhetischen Konventionen zuzurechnen, welche den spezifischen Status von Literatur ausmachen, sondern auch all jene Regulative, welche direkt oder indirekt Einfluss auf die Entstehung, Verbreitung und Wahrnehmung lit. Erzeugnisse ausüben: Neben den beispielsweise die Herausbildung eines lit. $/$ Kanons oder die Werturteile der $/ \mathrm{Li}$ teraturkritik legitimierenden ästhetischen Standards wären hier zur Rechtfertigung staatlicher Eingriffe (etwa gezielter $/$ Zensur-Maßnahmen) oder kirchlicher Sanktionen dienende politische, religiöse und moralische Normen, das Verhältnis zwischen verschiedenen Akteu- 
ren des lit. Marktes regulierende rechtliche Vorschriften (z. B. im >Urheberrecht), primär finanziellen Interessen dienende ökonomische Vorgaben (z. B. Buchpreisbindung) oder den spezifischen Status von Literatur und Literaten begründende gesellschaftliche Werte zu nennen.

Eine Unterscheidung zwischen genuin auf das Literatursystem bezogenen Normen und einem jenseits davon zum Tragen kommenden Normensystem erscheint wenig sinnvoll, da L.I. keinen autonomen Bereich konstituieren, sondern auf komplexe Weise mit anderen Bereichen lebensweltlicher Erfahrung interagieren. Ebenso ist es unangemessen, mit Blick auf L. I. zwischen Normen und institutionellen Trägern eine scharfe Trennlinie zu ziehen, sind die das Literatursystem prägenden Normen und die es ermöglichenden Strukturen doch aufs engste miteinander verknüpft.

L. I. bilden demnach keinen eigengesetzlichen Bereich, der sich gesellschaftlicher Einflussnahme entzieht, sondern sind immer abhängig von außerlit. Handlungsfeldern: Politische (Zensur, nationale Kulturpolitik), ökonomische, gesellschaftliche (soziale Stratifikation, Bildungsmöglichkeiten) oder im engeren Sinn kulturelle (vorherrschende Wertvorstellungen, religiöse Normen) Determinanten bleiben nicht ohne Wirkung auf L. I.; die Handlungen der Akteure im Bereich der Literatur haben grundsätzlich sozialen Charakter. Damit erweist sich die Vorstellung der Literatur als eines gleichermaßen eigenständigen und zweckfreien Bereichs kultureller Praxis als äußerst problematisch.

Dasselbe gilt für die Auffassung, der für die Evolution des lit. Kommunikationssystems fundamentale Wandel von Normen und Wertvorstellungen ließe sich allein aus literaturimmanenten Entwicklungen erklären. Literatur als gesellschaftliches Handlungssystem funktioniert nicht losgelöst von historisch spezifischen Rahmenbedingungen und wandelt sich mit ihnen; die Auseinandersetzung mit L.I. bedeutet deshalb immer auch, nach den gesellschaftlichen Bedingungen lit. Konzepte und Praktiken sowie deren institutionellen Verflechtungen zu fragen. In seiner Theorie des lit. Feldes hat Pierre Bourdieu deshalb die für das System der lit. Kommunikation im nzl. Europa konstitutiven Prozesse einer Ausdifferenzierung, einer Professionalisierung und eines Geltungsgewinns der L.I. zwar als eine fortschreitende Autonomisierung des lit. Kommunikationssystems gegenüber religiösen, politischen und ökonomischen Kräften beschrieben [2], zugleich jedoch in seinem wegweisenden Aufsatz Le marché des biens symboliques (1971/72; "Der Markt der symbolischen Güter $)$ die determinierende Funktion gesellschaftlicher Rahmenbedingungen für die Produktion und Zirkulation symbolischer Güter herausgestellt, zu denen er auch die Literatur zählt [1].

Im Zentrum der Beschäftigung mit L.I. steht hier demzufolge die in ständigem Wandel begriffene Wech- selbeziehung zwischen $\boldsymbol{\gamma}$ Literatur und $r$ Gesellschaft: $\mathrm{Zu}$ beschreiben gilt es dabei zum einen die institutionellen Rahmenbedingungen lit. Kommunikation und das dafür kennzeichnende System von Interdependenzen sowie zum anderen die auf das Literatursystem einwirkenden und dessen histor. Dynamik begründenden politischen, ökonomischen und sozialen Prozesse. Konkret heißt dies, dass sich das Augenmerk zunächst auf die verschiedenen historisch manifesten Akteure des lit. Systems etwa $r$ Autoren, $r$ Verlage oder $r$ Leser - und die von diesen Akteuren ausgeübten Praktiken richtet; die Texte treten demgegenüber in den Hintergrund. Ungeachtet ihrer zentralen Rolle innerhalb des lit. Systems werden also einzelne 7 Druckmedien ebenso wenig in den Blick genommen wie lit. 7 Gattungen, obwohl Letztere in der Forschung von einigen Autoren den L.I. zugerechnet worden sind [9]. Auf die systematisch angelegte Erfassung der L.I. im Modus der Aufzählung (s.u. 3.) folgt dann in einem zweiten Schritt die Erörterung ihres Zusammenspiels mit dem Ziel, das Literatursystem und die außerlit. Funktionsbereiche gesellschaftlichen Lebens als histor. variablen Zusammenhang zu erhellen (s.u. 4.). Zu weiterer Literatur vgl. die Bibliographien der einzelnen Verweisstichwörter.

\section{Konstituenten des Literatursystems}

3.1. Bereiche

3.2. Produktion

3.3. Distribution

3.4. Vermittlung

3.5. Rezeption

\subsection{Bereiche}

Kennzeichnend für das Literatursystem, wie es sich im nzl. Europa herausbildete, ist eine Vielzahl von I., die im Sinne einer funktionalen Differenzierung der Produktion (s.u. 3.2.), der Distribution (s. u. 3.3.), der Vermittlung (s.u. 3.4.) und der Rezeption (s.u. 3.5.) von Literatur zugeordnet werden können. Eine klare Trennung der einzelnen Funktionsebenen ist allerdings ebenso wenig möglich wie die konsequente Unterscheidung zwischen den Handlungsbereichen "lit. Text" und wnichtlit. Text " (Sachliteratur, Publizistik usw.), da diese nicht seiten gemeinsam an den I. des Literatursystems teilhaben; dennoch ermōglicht es die folgende Systematik, die mit Blick auf die nzl. europ. Verhältnisse wesentlichen Akteure und Praktiken lit. Kommunikation in ihrer Bedeutung für das gesamte Feld der Literatur zu benennen.

\subsection{Produktion}

Im Zentrum lit. Produktion stehen zunächst und erstens der aus eigenem Antrieb oder - seltener - für 
einen 7 Auftraggeber tätige 7 Autor einerseits und die verschiedenen Organisationsformen von Autorschaft andererseits. Bedeutsam ist mit Blick auf Ersteren die Frage, wie Autorschaft konzeptionalisiert bzw. welche Rolle dem Urheber eines lit. Textes jeweils zugewiesen wird. Nicht zufällig indizieren die unterschiedlichen Bezeichnungen für Verfasserschaft zugleich eine unterschiedliche Auffassung dessen, was einen Autor auszeichnet: Steht $T_{m \text { Dichter } ~}$ in der Regel für den Urheber eines poetischen Werks mit hohem ästhetischen Anspruch, so beinhaltet »Autor « keine Festlegung auf eine bestimmte Textkategorie. Die in der Praxis nicht konsequent gehandhabte Unterscheidung zwischen Dichter (lat. poeta) und Autor (lat. auctor) begegnet bereits im 16. und 17. Jh., gewann jedoch im Zuge der Durchsetzung der $r$ Autonomieästhetik seit dem späten 18 . Jh. an Bedeutung. Im Zusammenhang mit dem Autor ist übrigens auch der Übersetzer zu nennen (rÜbersetzung), der die Textgestalt des von ihm in eine andere Sprache übertragenen Werks in hohem Maße verantwortet und im Umgang mit dem Original bisweilen bemerkenswerte Eigenständigkeit aufweist.

Sehr früh begannen Autoren damit, sich als Kollektive zu formieren, um damit eine engere Vernetzung mit anderen lit. Tätigen, eine konsequentere Pflege volkssprachlicher Literatur oder eine Verbesserung ihres Status als Verfasser zu erreichen. Die im Milieu städtischer $/$ Handwerker angesiedelten Zusammenschlüsse der Meistersinger führen ins 16. Jh. zurück (7Meistersang); im 17. Jh. dominierten 7 Sprachgesellschaften wie die modellbildende Florentiner Accademia della Crusca (gegr. 1583), die Fruchtbringende Gesellschaft (gegr. 1617; vgl. 7 Korporation, Abb. 2) oder die Académie française (gegr. 1635), die zwar in ihren Satzungen als primäres Ziel die Pflege der jeweiligen $/$ Volkssprachen definierten, jedoch zugleich wirkungsmächtige Katalysatoren vernakulärer (volkssprachlicher) Dichtung darsteltten ( $/$ Akademie). Daneben existierten bereits seit dem 17. Jh. und dann vermehrt im 18. und 19. Jh. eine Reihe bisweilen eher locker organisierter, meist einem emphatisch gefeierten Freundschaftskult verpflichteter Gruppierungen (wie z. B. der Göttinger Hain; 7 Dichterbund), welche hinsichtlich ihrer sozialen Zusammensetzung und ihrer lit. Präferenzen auffällig homogen erscheinen.

Gemeinsam ist den in der Nz. nachweisbaren Autorenvereinigungen, dass sie sich - im Unterschied zu modernen Schriftstellerverbänden - weniger als auf die Durchsetzung ökonomischer und juristischer Standards bedachte berufsständische Organisationen verstanden, sondern vielmehr die Förderung der Literatur intendierten, die ihnen als kulturelle Praxis von besonderer Dignität galt. Zwar war den bis weit ins 19. Jh. meist nebenberuflich als Autor tätigen Literaten, die für ihre Werke im besten Falle ein 7 Honorar erhielten und auch nach der Einführung eines $/$ Urheberrechts nur in Ausnahmefällen ein existenzsicherndes Einkommen erzielten, durchaus bewusst, wie begrenzt die Möglichkeiten waren, aus lit. Schaffen ökonomisches Kapital zu schlagen; da sie sich als Autoren jedoch v. a. soziale und kulturelle Anerkennung erhofften, wog die Förderung durch einen hochrangigen 7 Mäzen oder die Krönung zum Dichter meist schwerer als pekuniärer Erfolg (vgl. $>$ Dichter 5.). Allerdings profitierten Autoren durchaus nicht nur von symbolischen Gratifikationen. Das Verfassen und die gezielte Widmung lit. Werke ebneten bisweilen den Weg in gut bezahlte höfische 7 Ämter und verhalfen auf diese Weise auch zu materiellem Gewinn.

Der Ebene der Produktion sind zweitens auch jene Infrastrukturen zuzurechnen, die der Veröffentlichung lit. Texte dienten, also die I. des Druckergewerbes ( 7 Druckmedien). An erster Stelle sind hier die Drucker selbst zu nennen, die v.a. im 16. und 17. Jh. den Druck von Schriften entweder als Nebenerwerb betrieben oder aber, v. a. in den bedeutenderen $>$ Residenzstädten, $\nearrow$ Reichsstädten und $T$ Universitätsstädten, große und technisch gut ausgestattete 7 Offizinen einrichteten, die unter $/$ Gelehrten und $>$ Bücher-Sammlern einen ausgezeichneten Ruf genossen.

Insbes. in den größeren Werkstätten erfolgte die Herstellung einer Druckschrift arbeitsteilig: Neben dem Schriftgießer waren hier der Setzer, der für die Bildvorlagen zuständige Zeichner, der Holzschneider oder Kupferstecher sowie der Drucker tätig; im Zuge der Professionalisierung des $/$ Buchdrucks und der Herausbildung neuer Illustrationstechniken seit dem späten 18. Jh. gesellten sich der Korrektor und der Lithograph dazu ( $/$ Druckgraphik). Schließlich versah der $/$ Buchbinder im Auftrag einer Offizin (vgl. / Informationsmedien, Abb. 1), häufiger jedoch eines Käufers der in der Regel in Form ungebundener Bogen verkauften Druckwerke Bücher mit einem Einband. Für die frühe Druckproduktion des 15. und 16. Jh.s gilt übrigens, dass eine Offizin sich kaum je auf in engerem Sinne lit. Werke beschränkte; auch mit Blick auf das 18. und 19. Jh., als die Drucker immer mehr dazu übergingen, Schriften nicht mehr in eigener Regie herzustellen und zu vertreiben, sondern sie im Auftrag von Verlegern zu produzieren ( $/$ Verlag), ist festzuhalten, dass Druckereien in der Regel Texte unterschiedlichen Charakters auf den Markt brachten, um profitabel zu sein.

\subsection{Distribution}

Dass der 7 Buchdruck sich innerhalb kürzester Zeit über ganz Europa ausbreitete, hängt wesentlich damit zusammen, dass er es ermöglichte, ein Werk in hoher Auflage herzustellen, und damit ökonomischen Gewinn versprach. Um die stetig wachsende Zahl von Druck- 
schriften abzusetzen, bedurfte es leistungsfähiger Vertriebsstrukturen, die sich daher auch parallel zum Siegeszug des Buchdrucks entwickelten. Eine wichtige Rolle kommt in diesem Zusammenhang der Frankfurter und der Leipziger 7 Buchmesse $\mathrm{zu}$, die - dies gilt insbes. für die bis ins 17. Jh. hinein bedeutendere Frankfurter Messe - Buchhändler aus ganz Europa anzogen und seit dem 15. Jh. als wichtiger Umschlagsplatz für gedruckte Literatur fungierten.

Es war jedoch v. a. der $\gg$ Buchhandel, der den Verkauf gedruckter $>$ Bücher an potentielle Leser gewährleistete: Da Herstellung, Vertrieb und Verkauf von Druckschriften noch bis ins 18. Jh. hinein nicht in getrennten Bahnen verliefen, waren es zunächst die Drucker selbst bzw. in deren Auftrag oder aber in eigener Regie agierende Kolporteure bzw. mobile Buchführer, die mittels eines immer größere Bereiche Europas umspannenden Vertriebsnetzes dafür sorgten, dass an Literatur Interessierte Druckerzeugnisse erwerben konnten. Seit dem 18. Jh. konzentrierte sich der Buchhandel zunehmend in festen Ladengeschäften, die ihren Kunden ein wachsendes Sortiment zur Verfügung stellten, das sie über verschiedene $T$ Verlage bezogen. Als Verleger betätigten sich in der Frühzeit des Buchdrucks in der Regel die Drucker selbst. Erst mit der im 18. Jh. vergleichsweise spät erfolgten endgültigen Trennung der Bereiche Herstellung, Vertrieb und Verkauf entstanden eigenständige Verlagsunternehmen, die für das von ihnen bestimmte Programm verantwortlich zeichneten sowie Druck und Verkauf von Literatur ausgliederten.

An der Verfügbarmachung gedruckter Literatur waren nicht nur die genannten gewerblichen Betriebe beteiligt, sondern auch die $/$ Bibliotheken. Neben den bereits im MA existierenden $\boldsymbol{\gamma}$ Kloster- und $\boldsymbol{\gamma}$ UniversitätsBibliotheken gewannen im Zuge von 7 Renaissance und 7Humanismus ab dem 16. Jh. fürstliche Privatbibliotheken und $\rtimes$ Gelehrten-Bibliotheken zunehmend an Bedeutung; außerdem wurden in urbanen Zentren vermehrt Rats- oder Stadtbibliotheken eingerichtet. Im Unterschied $\mathrm{zu}$ anderen Bibliothekstypen, deren Bestand in der Regel v. a. geistliche und gelehrte bzw. Sachliteratur umfasste, fand sich in den Privatbibliotheken des Adels nicht selten auch eine größere Zahl belletristischer Werke, die bis weit ins 18 . Jh. hinein noch einen vergleichsweise kleinen Teil der Druckproduktion ausmachten.

Einem seit dem späten 18. Jh. gesteigerten Interesse an sschöner " Literatur trugen die um 1800 florierenden kommerziellen Leihbibliotheken Rechnung, die es v.a. einem bürgerlichen Publikum ermöglichten, lit. Neuerscheinungen zu lesen, ohne jedes Mal den hohen Preis für ein Buch aufbringen zu müssen. Deren Niedergang hing nicht zuletzt damit zusammen, dass seit der Mitte des 19. Jh.s nicht nur der Buchpreis kontinuierlich sank, sondern zugleich die Zahl der öffentlichen, für die Benutzer meist kostenlosen Bibliotheken zunahm, was schließlich zu einer Verdrängung der kommerziellen Bibliotheken führte.

\subsection{Vermittlung}

Die in der Forschung zu den Funktionsweisen des lit. Kommunikationssystems häufig begegnende Trias von Produktion, Distribution und Rezeption klammert einen Bereich aus, der für den Status von Literatur in einer Gesellschaft von erheblicher Bedeutung ist: die Vermittlung. Damit sind hier jene I. und damit in Verbindung stehenden Praktiken gemeint, die darauf zielen, die als verbindlich erachteten Normen lit. Produktion, die lit. Texte selbst oder auch deren Urheber einem bisweilen ausgewählten, häufiger jedoch tendenziell breiten Adressatenkreis bekannt zu machen. Vermittlung von Literatur setzt immer voraus, dass die zu vermittelnden Texte bereits einer ersten Lektüre unterzogen wurden, in der Regel durch "professionelle" Leser wie z. B. Kritiker, Wissenschaftler oder Pädagogen; die auf dieser Lektüre basierenden Vermittlungsakte (u. a. TRezensionen) sind folgerichtig das Ergebnis eines Selektions-, Ordnungs- und Wertungsaktes, der sich individuellen, aber auch kollektiven Prägungen und Präferenzen der jeweiligen Vermittler verdankt. Prozesse lit. Vermittlung steuern demnach in hohem Maße sowohl die Produktion als auch die Rezeption von Literatur, sind sie es doch, die darüber bestimmen, welchen (nicht nur) ästhetischen Normen lit. Texte verpflichtet zu sein haben.

Bedeutung kommt in diesem Zusammenhang zunächst der $/$ Literaturtheorie und der $>$ Literaturkritik zu. Erstere stellt in normativer Absicht ein Modell poetischen Schreibens zur Verfügung, das Aussagen über die spezifischen Formen, Funktionen und Wirkungen von Dichtung enthält und den Autoren als Anleitung dienen soll. Zugleich stelt die 7 Poetik Wertungsmaßstäbe auf, die es den zeitgenössischen Rezensenten lit. Texte ermöglichen, ihr Urteil zu autorisieren. Beide, Literaturtheorie und Literaturkritik, leisten auf diese Weise einen entscheidenden Beitrag zur Herausbildung lit. /Kanones, indem sie ein Instrumentarium bereitstellen bzw. anwenden, das es erlaubt, Texte aufgrund der ihnen zugesprochenen ästhetischen Qualität zu klassifizieren. An der Entwicklung, Begründung und Propagierung lit. Kanones hat außerdem die Literaturwissenschaft bzw. die $/$ Literaturgeschichtsschreibung nicht geringen Anteil, da sie darüber entscheidet, welche Texte als erinnerungswürdig zu gelten haben und wo der jeweilige Text innerhalb eines regional, national, europäisch oder auch universal definierten kollektiven 7 Gedächtnisses zu verorten ist. Zugleich beteiligt sich die Literaturwissen- 
schaft an der Bereitstellung von Texten, indem sie sie ediert und damit ihre als endgültig postulierte Form bestimmt ( $/$ Edition).

Der Verfügbarmachung von Literatur dient auch das Literaturarchiv, dessen primäre Aufgabe darin besteht, Quellen zur Literatur (Handschriften, Dokumente, Drucke) zu sammeln, ordnen, bewahren und verzeichnen, um deren Zugänglichkeit zu gewährleisten. Zwar sind in fürstlichen und Universitätsbibliotheken handschriftliche Nachlässe aus dem 16. bis 18. Jh. überliefert, öffentliche 7 Archive mit klar umrissenem Sammelauftrag wurden jedoch v.a. seit der Mitte des 19. Jh.s eingerichtet und dienten von Beginn an auch als Orte, an denen an Autoren erinnert werden sollte. In dieser Funktion nähern sie sich den seit dem späten 19. Jh. gegründeten Dichtergedenkstätten (Dichtermuseen) an, die meist die Erinnerung an einen einzelnen Autor befördern sollen, indem sie dessen Leben und Werk einem interessierten Publikum vor Augen führen. Als Teil lit. Memorialkultur haben nicht zuletzt auch all jene Dichterdenkmäler etwa diejenigen zu Ehren Dante Alighieris in zahlreichen ital. Städten oder das Goethe-Schiller-Denkmal in Weimar (1857) - zu gelten, die im Zuge einer seit dem 19. Jh. zunehmend national ausgerichteten Kulturpolitik in den öffentlichen Raum Eingang fanden.

Der Vermittlung von Literatur dient schließlich auch der schulische Literaturunterricht (/Schule). Zwar hat sich im niederen Schulwesen der Literaturunterricht in den meisten europ. Staaten erst seit dem späten 19. Jh. etabliert; die schulische Beschäftigung mit lit. Texten reicht jedoch viel weiter zurück: War es zunächst v.a. der humanistischen Prinzipien verpflichtete gymnasiale \Latein-Unterricht ( $/$ Lateinschule), der Schülern der Frühen Nz. die Auseinandersetzung mit rhetorisch-poetologisch exemplarischer Dichtung ermöglichte, wurden seit dem 17. Th. verstärkt auch volkssprachliche Exempla in die gymnasiale Lehre integriert. Im Bereich des muttersprachlichen Leseunterrichts wiederum gewann Literatur an Bedeutung, seit $/$ Katechismus und $/$ Bibel ab der zweiten Hälfte des 18 . Jh.s als schulischer Lesestoff durch Lesebücher ergänzt und später verdrängt wurden, die eine Auswahl von für Schüler geeigneten lit. Texten enthielten.

\subsection{Rezeption}

Produktion, Distribution und Vermittlung von Literatur zielen letztlich immer auf deren Rezeption, und so kommt der individuellen und kollektiven Wahrnehmung lit. Texte grundlegende Bedeutung zu. Ihre häufigste Form dürfte die individuelle Lektüre sein, als deren Akteur der einzelne $/$ Leser im Mittelpunkt steht. Die individuelle Aneignung von Literatur verband sich jedoch seit jeher mit kollektiven Rezeptionsweisen, die meist an einen spezifischen lit. Kommunikationsraum etwa 7 Hof oder 7 Stadt - gebunden waren. Insbes. der Hof fungierte in der Frühzeit des Buchdrucks als wichtiges Zentrum lit. Austauschs, das erst seit dem späten 17. Jh. zugunsten einer zunehmend bürgerlich geprägten 7 Lesekultur an Bedeutung zu verlieren begann.

Die Institutionalisierung kollektiver Rezeptionsweisen von Literatur vollzog sich im Zusammenhang mit der Entstehung einer Reihe neuartiger Vergesellschaftsformen, deren zentrale Intention in der Förderung der individuellen oder auch gemeinschaftlichen Lektüre und v. a. im Gespräch über das Gelesene bestand. Eine frühe Form kollektiver Aneignung und Auseinandersetzung mit Literatur war der - meist von einer hochgestellten und gebildeten Frau geführte - lit. 7Salon, dessen Anfänge im Frankreich des 17. Jh.s zu suchen sind. Ungeachtet seiner zunächst engen Bindung an Hof und Adelskultur steht der Salon für eine TGeselligkeits-Form, in der durch soziale Zugehörigkeit oder Geschlecht begründete Hierarchien vorübergehend zugunsten eines Ideals geistig-künstlerischer $>$ Kommunikation zwischen gebildeten Männern und Frauen aufgehoben schienen. Literatur spielte dabei eine zentrale Rolle, entwickelten sich die europ. Salons doch bald zu einem bevorzugten Ort des Diskurses über aktuelle lit. Erscheinungen.

Einen in der Regel männlich dominierten Raum lit. Kommunikation stellten demgegenüber die seit der zweiten Hälfte des 18. Jh.s zunehmend verbreiteten $/ L e-$ segesellschaften dar. Die von ihnen eingerichteten Lesekabinette hielten für die Mitglieder zwar v.a. (populär-)wiss. 7 Zeitschriften sowie 7 Enzyklopädien bereit, waren jedoch auch ein Ort, an dem zeitgenössische Entwicklungen im Bereich der Literatur diskutiert werden konnten. In weit höherem Maße widmeten sich schließlich jene lit. Gesellschaften der mschönen « Literatur, welche seit dem 19. Jh. v.a. der Pflege des Werks eines einzelnen Autors dienten (Marbacher Schillerverein, 1835; Dt. Shakespeare-Gesellschaft, 1864; 7 Sprachgesellschaften).

Individuelle und v.a. kollektive Literaturrezeption erfolgte allerdings nicht nur im Rahmen der genannten I., sondern auch in geselligen Kontexten, die nicht in erster Linie auf Literatur zentriert waren. $\mathrm{Zu}$ erwähnen wären hier das 7 Theater, das als Aufführungsort musikalischer Anlässe (TOper; $r$ Ballett) das Publikum mit einem reichhaltigen Programm lockte, jedoch zugleich über eine $/ B$ Bühne verfügte, auf der lit. Werke, 7 Dramen, inszeniert werden konnten, sowie das 7 Kaffeehaus, das primär dem 7 Konsum von Getränken und Speisen, der rZeitungs-Lektüre und dem $/$ Spiel diente, das jedoch, nachdem es seit dem 18. Jh. zum Treffpunkt von /Intellektuellen und Künstlern avanciert war, zugleich Raum für lit. Schaffen und lit. Debatten bot. 


\section{Historische Entwicklung}

4-1. Übergeordnete Zusammenhänge

4.2. Renaissance, Humanismus, Reformation

4.3. Barock

4-4. Aufklärung

4.5. wZeitalter der Revolutionen « (1789-1848)

\subsection{Obergeordnete Zusammenhänge}

Die bisher genannten I. waren zwar eigenständige Akteure in der lit. Kommunikation, ihre spezifische Form und Funktion resultierte jedoch wesentlich aus ihrem Status innerhalb eines übergeordneten lit. Kommunikationszusammenhangs. Sie sind deshalb nicht isoliert zu betrachten, sondern als Elemente eines eng vernetzten, historisch variablen Systems: So führten etwa bestimmte Organisationsformen von Autorschaft (etwa die frühnzl. Sprachgesellschaften, welche die Mitgliedschaft an lit. Tätigkeit banden) zu einer verstärkten Produktion lit. Texte und trugen somit dazu bei, den lit. Markt zu beflügeln; die Professionalisierung des Vertriebssystems von Literatur und die Einrichtung öffentlicher 7 Bibliotheken befriedigten und nährten zugleich ein stetig wachsendes Bedürfnis nach Lektüre; die Herausbildung neuer poetologischer Normen wirkte sich auf den lit. TKanon und die wiss. Beschäftigung mit Literatur aus; und der technische Fortschritt im Buchdruck, der u. a. zu einer spürbaren Verbilligung lit. Publikationen führten, ebneten dem modernen lit. Massenmarkt den Weg.

Die Evolution des lit. Feldes in der Nz. war allerdings nicht nur die Folge innerlit. Dynamiken, sondern verdankte sich überdies und in hohem Maße außerlit. Entwicklungen. Wie bereits erwähnt, bildet das Feld der lit. Kommunikation keine autonome Entität, sondern ist vielmehr mit anderen gesellschaftlichen Handlungsfeldern - $>$ Politik, 7 Wirtschaft, 7 Gesellschaft - durch ein dichtes Netz von Interdependenzen verbunden, die dessen historisch zu differenzierende Relevanz bestimmen. Anhand einer "signifikanten Reihe von Synchronschritten " [5. 83], mit Blick also auf zentrale 7 Epochen der nzl. Literaturgeschichte, sollen ausgewählte Entwicklungen eher angedeutet als ausgeführt werden. Dabei gilt es zu beachten, dass die nachfolgend benannten Prozesse nicht in allen europ. Literaturen zu beobachten sind und dass sie außerdem nicht überall gleichzeitig stattfanden.

\subsection{Renaissance, Humanismus, Reformation}

Kennzeichnend für 7 Renaissance, $T$ Humanismus und $/$ Reformation ist die Bedeutung der Tneulateinischen Dichtung, die erst im Zuge des verstärkten Aufkommens volkssprachlicher Literatur (zunächst in Italien und Frankreich, im 17. Jh. auch in Deutschland) an Geltung verlor ('Sprachen, Literatur). Angesichts der humanistischen Verankerung poetischen Schaffens dominierte im 16. und noch bis ins 18. Jh. die Vorstellung des 7 Autors als eines poeta doctus, eines gelehrten Verfassers von Schriften, als deren Vorbilder meist Werke antiker Autoren fungierten ( $/$ Antikerezeption). Nicht nur in der lat., auch in Bereich der volkssprachlichen Literatur machte sich der Einfluss der antiken Dichtung bzw. der diese in die Nz. vermittelnden humanistischen r Philologie bemerkbar; zugleich wirkte auch vereinzelt die volkssprachliche ma. Literatur nach, so etwa in der rTierdichtung (Reineke Fuchs) oder beim v.a. in der franz. Epik beliebten Melusinenstoff.

Die Dichtung des 16. und 17. Jh.s richtete sich in der Regel an gelehrte oder zumindest gebildete $\lambda$ Leser, meist aus dem gehobenen $>$ Bürgertum und dem $/$ Adel. Dies erscheint umso natürlicher, als die geringe ${ }^{\top} \mathrm{Alph}$ abetisierungs-Quote innerhalb der Bevölkerung im Verbund mit den vergleichsweise hohen Kosten für ein 7 Buch, das als /Luxus-Ware galt, die Zahl der potentiellen Rezipienten erheblich begrenzte. Erschwerten Zugang zu Literatur hatten insbes. die Bewohner ländlicher Gebiete, während in den 7 Städten, die v.a. im 16. Jh. die Zentren nicht nur des Buchdrucks, sondern der lit. Kommunikation überhaupt bildeten, bisweilen auch den Angehörigen des /Kleinbürgertums - etwa Handwerkern - die Auseinandersetzung mit lit. Werken möglich war.

Immerhin lassen sich mit Blick auf die Nz. verschiedene Modi oraler und semioraler Vermittlung lit. Tradition beschreiben, die vermuten lassen, dass auch innerhalb illiterater Kreise bestimmte Formen lit. basierter Kommunikation existierten ( $/$ Schrifflichkeit). Es waren insbes. die Reformatoren, die sich zunächst dafür einsetzten, dass durch individuelle $/$ Bibel-Lektüre, aber auch durch mündlich und performativ vermittelte lit. Manifestationen ( $/$ Kirchenlied; $/$ Schuldrama) größere Leser- bzw. Publikumskreise erschlossen wurden. Durch Indienstnahme der Literatur für religiöse Zwecke versuchten die Repräsentanten der protest. und in der Folge auch der kath. Kirche(n), eine nicht nur breitenwirksarne, sondern auch wirkungsmächtige Plattform für die konfessionelle Auseinandersetzung zu etablieren; im 16. Jh. überwogen neben Gattungen der 7 Lyrik daher auch das /Drama und die 7 Fabel, die dem didaktisch ausgerichteten Literaturkonzept v. a. geistlicher Autoren bes. affin erschienen.

Parallel zu den Kirchen, die danach strebten, Einfluss auf die lit. Kommunikation zu gewinnen, behauptete v.a. der Staat einen umfassenden Anspruch auf die Kontrolle der lit. Produktion, Distribution und Rezeption. Die Obrigkeiten bemühten sich denn bis ins 19. Jh. auch mit wechselndem Erfolg, mittels /Zensur-Bestimmungen Werke zu unterbinden, deren Inhalt als inopportun beurteilt wurde. 


\subsection{Barock}

Die Etablierung der volkssprachlichen europ. Literaturen und damit der barocken Kunstdichtung zu Lasten der neulat. Poesie erfolgte parallel zur Herausbildung absolutistischer politischer Strukturen in Europa ( $/ \mathrm{Ba}-$ rock; $/$ Absolutismus). Der humanistisch gebildete poeta doctus verkörperte zwar weiterhin den idealen Autor; dieser erfuhr jedoch im 17. Jh. in der Regel eine stärkere Anbindung an den $\mathbf{r H o f}$, der als lit. Zentrum den Städten zunehmend den Vorrang streitig machte. Nach der verfassungsrechtlichen Klärung u.a. der konfessionellen Verhältnisse in Europa durch den 7 Westfälischen Frieden (1648) löste sich die Dichtkunst insbes. in der zweiten Hälfte des 17. Jh.s immer stärker aus ihrer religiösen Bindung und stellte sich in den Dienst politischer, nicht selten höfischer Anliegen. Dies gilt etwa für die Lyrik ( $/$ Panegyrik), die 7 Tragödie, die nicht selten Fürstinnen und Fürsten auf die Bühne brachte, oder den theroischen Roman, der zum Medium politischer Reflexion avancierte.

Gleichzeitig sind auch lit. basierte, durch bestimmte Gelegenheiten (wie z. B. Geburtstag, Hochzeit oder Bestattung) induzierte Formen der Kommunikation zu beobachten, die sich in erster Linie innerhalb bürgerlicher Kreise entwickelten ( $/$ Gelegenheitsdichtung), denen mit Blick auf das lit. System grundsätzlich, nicht nur als Autoren, sondern auch als Vermittlern und Rezipienten weiterhin große Bedeutung zukam. Erfolgte die Verständigung über lit. Sachverhalte in den im 17. Jh. gebildeten, Aristokraten und Bürgerlichen gleichermaBen zugänglichen Dichter- und 7 Sprachgesellschaften noch standesübergreifend, so waren es meist gelehrte bürgerliche Verfasser (etwa Joseph Justus Scaliger oder Martin Opitz), denen wir nicht nur die überlieferten lit. Werke, sondern auch das poetischer Produktion zugrunde liegende ästhetische Normengefüge verdanken ( 7 Poetik).

Angesichts einer engeren Bindung der $/$ Literaturtheorie und -praxis an den Hof, angesichts einer weiterhin gültigen Auffassung vom Dichter als einem $7 \mathrm{Ge}$ lehrten, angesichts der immer noch sehr hohen Buchkosten sowie schließlich angesichts der geringen Fortschritte in der Alphabetisierung der Bevölkerung blieb lit. Kommunikation auch im sog. Barock das Privileg vergleichsweise kleiner Bevölkerungsgruppen, die überdies einer im Zuge der Modemisierung staatlicher $/$ Verwaltung zunehmend effektiveren Kontrolle durch die Zensur unterworfen waren.

\subsection{Aufklärung}

Eine auch in quantitativer Hinsicht signifikante Dynamisierung der lit. Kommunikation erfolgte erst im
18. Th. durch die 7 Aufklärung. Zum einen wandelte sich im Zuge der Herausbildung einer immer stärker bürgerlich geprägten 7 Öffentlichkeit die Rolle des Autors, welcher sich zunehmend als Erfüllungsgehilfe eines (volks-)pädagogischen Auftrags definierte, der auf eine grundlegende Umwandlung gesellschaftlicher Verhältnisse in aufgeklärtem Sinne zielte. Nicht zufällig waren es daher auch lehrhaft ausgerichtete $\pi$ Gattungen wie /Fabel, $\lambda$ Lehrdichtung, $\lambda$ Epigramm, $>$ Aphorismus oder rSatire, denen im 18. Jh. große Bedeutung zukam; daneben feierte auch der 7 Briefroman Erfolge, der insbes. unter Frauen Anklang fand, die neu als Autorinnen und v. a. als 7 Leserinnen in Erscheinung traten.

Die Erschließung neuer Leserkreise konzentrierte sich zunächst v.a. auf jene Schichten, die bereits im 16 . und 17. Jh. vorrangig zu den Rezipienten lit. Werke gehört hatten, und ist wesentlich der an Bedeutung gewinnenden, belletristische Werke bevorzugenden weiblichen Lektüre zu verdanken, die im späten 18 . Jh. einen ersten Expansionsschub des lit. 7 Buchmarkts begünstigte und damit eine spürbare Dynamisierung des Feldes der lit. Kommunikation bewirkte. Einen Beitrag zu dieser Dynamisierung leisteten einerseits neuartige 7 Druckmedien, insbes. die $/$ Zeitschrift, die im 18 . Jh. als wichtigstes Forum lit. Auseinandersetzung gelten darf, und der $\mathrm{A}$ Almanach - beide eröffneten einem breiteren Publikum den Zugang zu lit. Debatten -, sowie andererseits neuartige Formen organisierter Lektüre und geselligen Austauschs über Gelesenes, etwa rLesegesellschaften (vgl. Abb. 1) und v.a. die im 17. Jh. im Umfeld des Hofes entstandenen und seit dem 18. Jh. auch in bürgerlichen Kreisen beliebten 7 Salons. Dass seit dem 18. Jh. eine wachsende Zahl von Menschen an lit. Kommunikation partizipierte, ist nicht zuletzt den Bemühungen der Aufklärer um eine bessere schulische Bildung der Bevölkerung geschuldet, die zu einem Anstieg der Lesefähigkeit führten ( $/$ Lesekultur).

\section{5. "zeitalter der Revolutionen « (1789-1848)}

Der Entwicklung des lit. Kommunikationssystems zu einem Massenmarkt leisteten jedoch v. a. jene Prozesse Vorschub, die sich mit Blick auf den Zeitraum von 1789 bis 1848 - einem Zeitalter nicht nur politischer 7 Revolutionen - beschreiben lassen. Kennzeichnend ist in diesem Zusammenhang eine geänderte Auffassung des Autors, die - unter Berufung auf ein autonomieästhetisches Verständnis lit. Produktion (/ Autonomieästhetik) - eine Differenzierung zwischen $r$ Dichter und Schriftsteller impliziert. Stand der Dichter für einen autonomen lit. Bereich ästhetisch anspruchsvoller Poesie, mit dem sich sowohl neue lit. Kanones als auch die sich herausbildende Literaturwissenschaft legitimieren ließen, so verwies der Begriff „Schriftsteller « in der Regel 


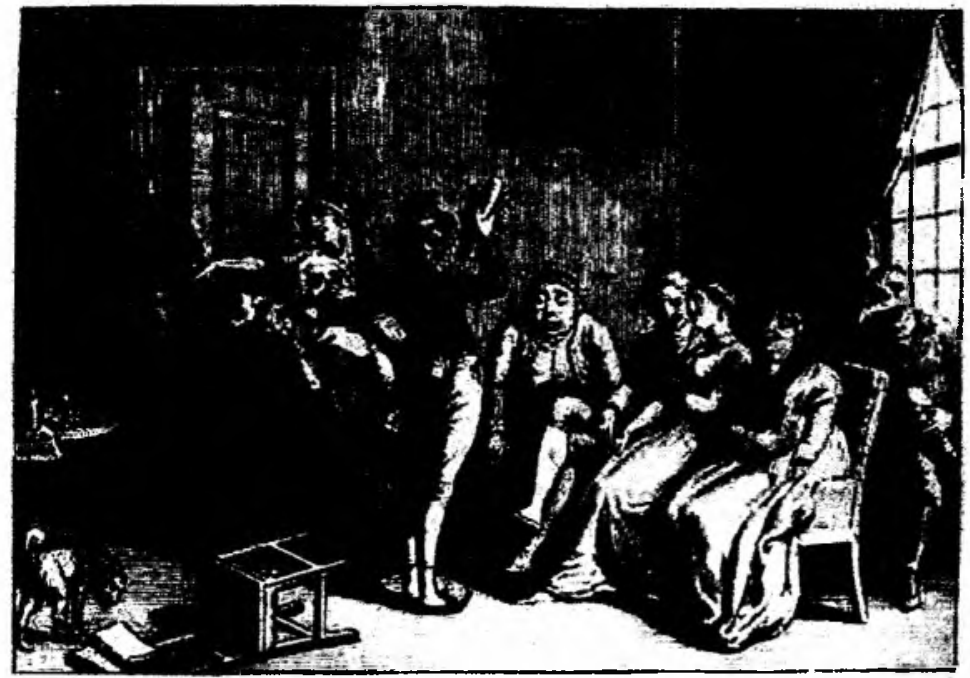

Abb. 1: I.H. Ramberg, Sentimentaler Leseconvent nach dem allerneuesten Zuschnitte (aus: C. F. Pockels, Miscellen für Freunde und Freundinnen des ernsten Nachdenkens ..., 1803. Bd. 2, Taf. 4). Die bürgerlichen Lesegesellschaften praktizierten und diskutierten zunächst ganz im Zeichen der Aufklärung kollektive Lektüre, wurden dann aber zu geselligen Vereinen. In dieser Karikatur liest ein selbst ernannter Dichter, der laut Original im »Odensturme den Stuhi umgestürzt" hat und sbeynahe den alten Professor Poesos et Eloquentiae ordinarius vom Stuhle wirft, vor einem Audito. rium von »Eingeweihten« (ergriffenen Damen) und "Pro fanen (gelangweilten Herren).

eher auf einen politischen Absichten verpflichteten oder aber den Gesetzen des Marktes gehorchenden Verfasser tendenziell anspruchsloser Werke für ein breiteres, lit. nur bedingt gebildetes Publikum.

Die Ausrichtung des zunehmend effizienter organisierten 7 Buchmarkts an den Bedürfnissen einer wachsenden Leserschaft begünstigte auch den Siegeszug des $\lambda$ Romans und der 7 Novelle, die in der Folge zu äußerst beliebten Gattungen avancierten und sich im Lauf des 19. Jh.s in zahlreiche Subgenres ausdifferenzierten. Sie bilden wichtige Konstituenten des modernen lit. Massenmarkts, der seit dem 19. Jh. das lit. Feld charakterisiert.

Zentrale Voraussetzung für die Genese und Etablierung eines lit. Massenmarktes bildeten zunächst jedoch eine Reihe von Innovationen im Bereich des Buchdrucks, der 7 Papier-Herstellung und der Buchbindetechnik (etwa die Erfindung der Schnellpresse 1812 oder die Entwicklung des Holzschliffverfahrens 1845), die insgesamt eine Beschleunigung und Verbilligung des Buchdrucks bewirkten. Zugleich zeichnete sich eine Professionalisierung der Distribution von Literatur $a b$, nicht zuletzt begünstigt durch ein besseres $/$ TransportSystem ( $/$ Eisenbahn) oder neuartige Absatzmodelle, wie etwa den Verkauf umfangreicher Werke in Teillieferungen ( 7 Subskription). Bedingt durch Bildungsreformen, insbes. die Einführung der Schulpflicht, stieg außerdem die Alphabetisierungsquote rapide an, was zur Folge hatte, dass nun auch breitere bäuerliche Bevölkerungsschichten oder die Arbeiterschaft als Rezipienten von Literatur in Betracht kamen. Die erhöhte Nachfrage wiederum trug $\mathrm{zu}$ einer Verbesserung der rechtlichen Stellung des Autors bei ( $\boldsymbol{T}$ Urheberrecht), dem die Er- träge seines Schreibens nun im besten Fall die Existenz als freier Schriftsteller ermöglichten.

Von Belang war außerdem die in den meisten europ. Staaten erst nach längerem Kampf gegen staatliche Zensureingriffe erwirkte 7 Presse- und Meinungsfreiheit, welche - nicht zuletzt durch den Abbau bürokratischer Hürden - die lit. Produktion begünstigte. Die bereits im 18. Th. einsetzende Entwicklung neuer Foren für lit. Austausch bzw. lit. Kritik wie etwa Salons oder Literaturzeitschriften schließlich fand im 19. Jh. ihre Fortsetzung; zugleich erfolgte, begünstigt durch den das europ. Staatensystem erfassenden Nationalisierungsschub, die Gründung von 7 Museen, Gedenkstätten und Archiven, welche die kollektive Erinnerung an die eigene, als "national» definierte Literatur sichern sollten.

\section{$\rightarrow$ Autor; Buchdruck; Buchhandel; Buchmarkt; Dichter; Lesegesellschaften; Lesekultur; Literatur; Literaturkritik; Literaturtheorie; Musik; Salon; Sprachgesellschaften}

[1] P. Bourdieu, Le marché des biens symboliques, in: L'année sociologique 22, 197-1972, 49-126 [2] P. Bourdieu, Les règles de l'art. Genèse et structure du champ littéraire, 1992 (dt. 1999) [3] G. Corsını, L'istituzione letteraria, 1974 [4] J. Dubois, L'institution de la littérature. Introduction à une sociologie, 1978 [5] G. GAISER, Literaturgeschichte und literarische Institutionen, 1993 [6] O. LoRENZ, Art. Literarisches Leben, in: RDL, 2, 2000, 438-441 [7] K.-S. Rehaerg, Ansätze zu einer perspektivischen Soziologie der Institutionen, 1973 [8] S.J. ScH MIDT, Grundriß der Empirischen Literaturwissenschaft, 1991

[9] W. VossKamP, Gattungen als literarisch-soziale Institutio nen, in: W. Hixcx (Hrsg), Textsortenlehre - Gattungs geschichte, 1977, 27-44. 